\title{
PET/MRI: Applications in Clinical Imaging
}

\author{
Karin Anna Herrmann • Andres A. Kohan • Maria Chiara Gaeta • \\ Christian Rubbert - Jose Luis Vercher-Conejero - Raj Mohan Paspulati • \\ Kalemis Antonis • Bahar Mansoori · Peter F. Faulhaber • Norbert Avril • \\ Pablo Riera Ros
}

Published online: 8 August 2013

(C) Springer Science+Business Media New York 2013

\begin{abstract}
PET/MRI is a new hybrid modality which is increasingly being used in clinical settings, although both clinical evaluation and technical optimization are still an ongoing process. Initial experience with this new imaging device proves promising for oncologic applications. Other clinical indications in the field of cardiac imaging and neuroimaging are also being explored. This article aims to review the current status of PET/MRI and its value in oncologic applications, and summarizes our own preliminary experience in this field.
\end{abstract}

Keywords $\mathrm{PET} \cdot \mathrm{MRI} \cdot \mathrm{PET} / \mathrm{MRI} \cdot$ Imaging

\section{Introduction}

Positron emission tomography (PET)/magnetic resonance imaging (MRI) is a new hybrid imaging modality combining two powerful diagnostic imaging tools. The individual strengths and weaknesses of these two imaging

K. A. Herrmann ( $₫)$ · A. A. Kohan - M. C. Gaeta · C. Rubbert ·

J. L. Vercher-Conejero · R. M. Paspulati · B. Mansoori ·

P. F. Faulhaber · N. Avril · P. R. Ros

Department of Radiology, University Hospitals Case Medical

Center and Case Western Reserve University, 11100 Euclid

Avenue, Cleveland, OH 44106, USA

e-mail: karin.herrmann@uhhospitals.org

A. A. Kohan

e-mail: andreskohan1@gmail.com

M. C. Gaeta

e-mail: mc.gaeta@gmail.com

C. Rubbert

e-mail: christian.rubbert@med.uni-duesseldorf.de

J. L. Vercher-Conejero

e-mail: jlvercher@hotmail.com modalities are believed to be synergistic and complementary in that the advantages of either component compensates for the limitations of the other. Thus, PET/MRI combines the highest anatomical detail as well as biochemical and functional information provided by MRI with the metabolic, molecular, and physiologic information from PET.

PET/MRI appeared in the clinical setting in 2007 [1, 2] with the first integrated PET/MRI scanner for brain applications. Since then it has undergone much technical development and optimization to make possible the coexistence of the two fundamentally different scanner technologies either adjacent to each other or in an integrated fashion. This combined imaging modality has now passed the level of an experimental research device and is increasingly being used clinically. Although most of the use of this new hybrid modality is still work in progress, the first clinical experience is now available for applications in oncology, and to a lesser extent for cardiac and neurologic applications. In general, the experience is

\author{
R. M. Paspulati \\ e-mail: raj.paspulati@uhhospitals.org \\ B. Mansoori \\ e-mail: bahar.mansoori@uhhospitals.org \\ P. F. Faulhaber \\ e-mail: peter.faulhaber@uhhospitals.org \\ N. Avril \\ e-mail: norbert.avril@uhhospitals.org \\ P. R. Ros \\ e-mail: pablo.ros@uhhospitals.org \\ K. Antonis \\ Philips Healthcare, Guildford, UK \\ e-mail: antonis.kalemis@philips.com
}


limited to the use of 2-deoxy-2-( $\left.{ }^{18} \mathrm{~F}\right)$ fluoro-D-glucose (FDG). Mainly in Europe there is a substantial preliminary experience with other tracers, including $\left[{ }^{18} \mathrm{~F}\right]$-fluorocholine, $\left[{ }^{11} \mathrm{C}\right]$ acetate, and $\left[{ }^{18} \mathrm{~F}\right]$-fluoroethyltyrosine as well as $\left[{ }^{11} \mathrm{C}\right]$-Pittsburgh compound $\mathrm{B}$ and $\left[{ }^{15} \mathrm{O}\right]$ - and $\left[{ }^{90} \mathrm{Y}\right]$-carrying tracers.

This article focuses on our initial clinical experience with applications of PET/MRI in oncologic imaging with FDG and highlights the potential incremental value of PET/MRI over current imaging standards based on these experiences and current literature.

To date, two fundamentally different scanner designs are commercially available: one is a PET detector inserted in the bore of an MRI system $[3,4 \bullet]$ and the other represents a sequential or tandem approach, where the PET scanner is located in the immediate vicinity of the MRI system with the patient couch placed between the two devices [5] or with a shuttle table between the two components [6-8]. Imaging protocols and workflow are dependent on the design of each device. The first approach allows simultaneous acquisition of PET and MRI data, whereas the tandem approach requires sequential data acquisition with the patient moving between the PET and MRI scanners while remaining in the exact same position on the scanner table. Both scanner designs operate with 3-T MRI systems; the PET scanner of the tandem device offers time-of-flight (TOF) PET technology. The TOF technology allows better control of the noise in the reconstructed image with direct improvements in the signal-to-noise ratio, resulting in higher effective scanner sensitivity [9]. As a result, TOF allows shorter acquisition times or less activity to be injected while maintaining image quality and quantification [10, 11].

Both PET/MRI systems provide specific MRI sequences for magnetic-resonance-based attenuation correction (MRAC) of PET data. Attenuation correction is required to account for the differences in photon absorption in different tissue of the human body, which is necessary to allow correct quantification of PET radiotracer activity. In PET/ computed tomography (CT) this information is obtained from the CT data, whereas in MRI the attenuation map has to be generated using these specific MRI sequences and segmentation models for body tissues such as air, lung, fat, soft tissue, and cortical bone. The accuracy of attenuation correction determines the accuracy of quantification of regional radiotracer activity in PET, which is often expressed as standardized uptake values (SUVs).

Unlike CT attenuation correction, MRAC cannot convert directly the MR images to attenuation maps as MRI does not provide electron density information, which is the primary cause of photon attenuation. In MRI, proton density is low in both air and cortical bone, and it is one of the challenges to correct for those tissues, which have very different attenuation characteristics. Currently available
MRAC methods differ between the different PET/MRI tomographs. Although the MRACs of both commercial systems are based on automated tissue segmentation, the two algorithms operate on different MRI sequences and segment different numbers of tissue classes. One MRAC is based on a two-point Dixon MRI sequence with segmentation of four tissue classes (air, lungs, soft tissue, and adipose tissue) [12], whereas the other operates on images acquired with a short flip angle T1-weighted (T1w) sequence and segments tissue into three classes (air, lungs, and soft tissue) [13]. Both approaches do not account for cortical bone, which is the highest-attenuating material in the human body. Current efforts in determining the performance of PET/MRI in oncologic imaging focus on the comparison between PET/CT with PET/MRI regarding lesion detection. In addition, relative tissue activity concentrations, or SUVs, of various tissues and organs were compared between PET/CT and PET/MRI [14, 15]. Since in PET/MRI the calculation of SUVs depends on the type of sequence used for attenuation correction and on the segmentation model applied, the accuracy of SUVs may differ between scanner designs. Initial experience has shown that for both scanner designs, SUVs of target lesions are generally similar between PET/CT and PET/MRI [4•, 16, 17]. However, for certain body parts and areas with either high or low tissue attenuation such as air and cortical bone, SUVs showed a larger variation between PET/CT and PET/MRI [14]. Lesions located in or adjacent to bone are subject to underestimation of PET tracer uptake by up to $11.2 \pm 5.4 \%$ (range $1.5-30.8 \%$ ) and $3.2 \pm 1.7 \%$ (range 0.2-4\%) depending on the segmentation algorithm used $[18,19]$. Current segmentation algorithms for whole body do not account for bone attenuation.

In our own experience with a Philips Ingenuity TF PET/ MR system, attenuation correction is performed using a whole-body T1w 3D gradient echo sequence and a threesegment model. Our experience encompasses 116 patients with oncologic diseases, who all underwent a doublescanning protocol of PET/CT followed by PET/MRI in one imaging session following one single injection of a standard activity of FDG. In almost all patients PET/CT was performed first approximately $60 \mathrm{~min}$ after tracer injection. PET/MRI followed the PET/CT scans with a transfer time of approximately 15-20 min. All patients were prospectively enrolled if FDG-PET/CT was clinically requested for primary staging or for follow-up restaging. All patients consented to participate in the study. Since in our setting the PET/CT and PET/MRI systems are both equipped with the same TOF PET technology and the same type of detectors, there is no technology-related difference in the PET part. The spectrum of diseases included primary cancer of various origins: lung, head and neck, lymphoma, melanoma, colorectal, breast, esophagus, pancreas, uterus 
and cervix, and other gynecologic tumors. Although PET/ MRI is valuable in neuro-oncology, discussion of brain neoplasms is beyond the scope of this review, which focuses on our oncologic experience outside the central nervous system.

\section{PET/MRI and Workflow}

A specific challenge in PET/MRI besides attenuation correction is to develop an optimized workflow. To combine two imaging modalities with fairly time intensive acquisition protocols for both MRI and PET requires a specific tailored design of the imaging protocols. It is important to avoid a lengthy overall examination so as to not compromise patient comfort and acceptance as well as image quality.

The imaging protocols and the workflow are dependent on the design and the type of the PET/MRI device. In integrated PET/MRI systems, the image acquisition of PET and MRI can occur simultaneously for a given bed position. In the sequential approach, MRI is performed either before or after PET data acquisition. Under certain circumstances, the uptake phase of the PET tracer can ideally be used for the MRI portion of the scan.

For both designs, workflow suggestions are now available [4•, 20• 21-23]. The fundamental decision in both instances is, however, if partial MRI, focused on an area or organ of interest, or whole-body MRI should be performed, or both. The extent and detail of an organ-based MRI protocol may overstrain a combined imaging protocol in one imaging session, especially if functional imaging including diffusion-weighted imaging (DWI) and dynamic contrast enhanced (DCE) imaging is also considered. Although most of the organ-based protocols may initially be similar to standard protocols for MRI-only examinations, the future will have to determine to what extent MRI can be condensed to be a "minimum necessary" protocol with maximum diagnostic outcome in view of the additional PET data.

Various approaches are possible to implement wholebody MRI in the setting of PET/MRI. The literature provides experience on whole-body MRI as a single modality examination. Those imaging protocols included T2weighted (T2w) and $\mathrm{T} 1 \mathrm{w}$ imaging with and without fat saturation [24, 25]. For the display of the entire body including the extremities, the coronal imaging plane is preferred. The examination time is approximately $15 \mathrm{~min}$ for one whole-body data set [25] and up to $40 \mathrm{~min}$ in other studies [26, 27].

The needs for whole-body imaging in the setting of PET/MRI are slightly different from the needs for wholebody stand-alone MRI in that image fusion with PET data will be desired and the acquisition time is a critical factor. Any cross-sectional data set can be fused with another, including MRI data from any acquired plane. However, to produce the highest quality of the fused images in each plane, 3D imaging sequences are preferable in order to be able to guarantee the same high image quality and detail in axial, sagittal, and coronal reconstructions. In this way, PET images can be directly fused and compared with MRI images. For this purpose, the 3D T1w two-point mDIXON sequence has demonstrated high value and numerous advantages [28].

The whole-body two-point mDIXON sequence generates a set of four different image qualities with different signal characteristics in one image acquisition; these are wateronly, fat-only, in-phase, and opposed-phase T1w images. Water-only images provide an excellent suppression of the signal of fat tissue, which can ideally be combined with postcontrast imaging. The in-phase and opposed-phase images allow the assessment of chemical shift effects, which are used to identify intravoxel fat and fatty infiltration. At the same time, $\mathrm{T} 1 \mathrm{w}$ in phase imaging is helpful in assessing bone marrow. All four signal characteristics are obtained in a data acquisition time of approximately $18 \mathrm{~s}$ per image stack and position. The sequence can be applied in a breath-hold examination and allows for isotropic voxel configuration. These features make the two-point mDIXON sequence well suited for PET/MRI in that it helps minimize the acquisition time while providing comprehensive diagnostic tissue information.

In our approach to the PET/MRI workflow, we decided to use a combination of whole-body imaging based on precontrast and postcontrast imaging using the mDIXON sequence complemented by an organ-based imaging protocol. Depending on the body region, a dedicated organbased protocol will be followed by a whole-body acquisition, which typically adds only a few minutes to the total examination time. Further details of the workflow will be discussed in the sections for each tumor category.

Head and Neck Cancers

Diagnosis and staging of head and neck cancers is a conjoint task of clinical examination, endoscopy with biopsy, histopathology, and imaging. Cross-sectional imaging techniques such as CT and MRI have proven their value in local staging and preoperative assessment of local tumor extent but show limitations in the correct assessment of nodal involvement. Although both CT and MRI as individual modality provide high sensitivities $(69-88 \%$ and 76-94\%, respectively), they are limited in specificity, ranging from 40 to $94 \%$ [27]. In comparison, PET/CT provides sensitivities and specificity between 87 and $94 \%$.

In a posttreatment setting after radiochemotherapy or surgery, both CT and MRI are limited by nonspecific 
appearances of posttherapeutic changes. The diagnostic challenge is the distinction between residual or recurrent disease from inflammatory changes or scar tissue formation [29]. PET and PET/CT have a well-established role in the assessment of local and distant metastatic disease of head and neck neoplasms, specifically in nodal staging, offering a high sensitivity and specificity $[30,31]$. However, in a posttreatment setting, PET has limitations similar to those of CT and MRI alone in distinguishing tumor from inflammatory tissue.

The value of software-based fusion of images from PET and MRI was shown by Nakamoto and Tamai [32]. The overall sensitivity of MRI increased from 67 to $92 \%$ with software-based fusion of PET and MRI images. Studies comparing the accuracy of co-registered PET/MRI images with that of fused image data sets are not yet available.

The challenges of PET/MRI in the head and neck regions are numerous and mostly technical in nature. Since the nasopharynx, oropharynx, and hypopharynx are anatomic regions at interfaces between bone, air, and soft tissue, correct tissue attenuation correction is challenging. T1w 3D gradient echo sequences do not distinguish between bone and soft tissue; hence, SUV measurements in these areas tend to be inaccurate. Dedicated MRI sequences have been proposed such as ultrashort echo time, ultrashort echo time double echo, and even ultrashort echo time triple echo sequences, which are able to distinguish air, bone and soft tissue in MRI and therefore allow their correct segmentation [33].

Another important issue is the precise positioning of the head and neck during sequential scanning between PET and MRI in the tandem PET/MRI design. Positioning aids help to sufficiently immobilize the head and neck for that purpose and help avoid positioning errors. This issue might be of less significance in the simultaneous acquisition.

An additional factor is the presence of dental metal implants, which have a significant impact on MRI images since they produce areas of signal void to a variable and unpredictable degree [22]. These MRI signal voids are not individually corrected prior to the generation of an attenuation map. As such, however, they may be a source for the generation of defective attenuation maps and failed segmentation for the respective tissue components depending on the underlying segmentation algorithm. Their impact on correct SUV measurements has not been studied in detail but raises concerns of being, again, challenging.

The workflow does not pose specific problems in head and neck cancers. In an integrated device with simultaneous image acquisition for PET and MRI, a head-andneck protocol can be obtained in $35 \mathrm{~min}$, including a whole-body scan for attenuation correction and anatomic reference of FDG-avid lesions with a two-point mDIXON sequence [34].
In a sequential approach with the tandem-designed PET/ MRI, the clinical morphologic MRI protocol can be performed during the PET tracer uptake time. Our comprehensive protocol including local morphologic imaging and whole-body anatomic reference for the PET information with a two-point mDIXON and T2w single-shot or turbo spin echo sequences takes approximately 25-30 min, with an additional 10-15-min period for the PET acquisition.

Current literature on PET/MRI in head and neck cancers is limited to the initial experience reported by Platzek et al. [22] in 20 patients. They found that PET/MRI of the whole headand-neck region is feasible with a whole-body PET/MRI system without compromising the PET or MRI image quality. The PET/MRI system in comparison with a PET-only device yielded higher SUVs in FDG-avid lesions and PET/MRI detected more relevant lesions in the head-and-neck area. Some of the SUV and detection differences likely reflected a long delay between the PET and PET/MRI of 113 min on average as well as PET scanner differences. No reference standard was provided to prove malignancy in either PETpositive or MRI-positive lymph nodes that were additionally detected with PET/MRI. In the same work, the authors reported a total acquisition time of $39 \mathrm{~min}$ for PET/MRI.

In our own experience, PET/MRI has a performance similar to that of PET/CT in the nodal staging of head and neck cancers. We analyzed with an independent multireader study design 14 patients with head and neck neoplasms who underwent both PET/CT and PET/MRI in one imaging session following a single injection of FDG. The average delay between the two scans was $56 \mathrm{~min}$. Of 105 FDG-avid lesions identified with PET/CT, $80 \%$ were correctly identified and exactly matched in PET/MRI. In 11 of 14 patients $(79 \%)$, there was complete agreement in staging between observers. In the cases with discrepant findings, PET/MRI found two additional lymph nodes per patient (unpublished data).

The expected potential benefit of PET/MRI rests in the exact superimposition and, therefore, precise anatomic correlation between FDG-avid tissue and the anatomic detail derived from MRI images. This might improve not only the TNM staging of the tumor but also the assessment of postoperative or post-radiation-therapy changes.

The value of functional information from MRI, particularly of DWI, as a prognostic tool in head and neck cancer is well known [35, 36]. Correlations of DWI with PET information and tracer uptake and the value of the two conjoint modalities remain to be investigated.

\section{Lung Cancer}

To date, lung cancer staging and follow-up is the domain of $\mathrm{PET} / \mathrm{CT}$, which provides very high accuracy for local $\mathrm{T}$ and $\mathrm{N}$ and distal $\mathrm{M}$ staging [37]. For the assessment of 
brain metastases, it is typically combined with separate MRI of the brain. Given the outstanding performance of FDG-PET/CT in lung cancer staging, one may question the need for comparing PET/CT with PET/MRI since MRI has well-known limitations in the detection of pulmonary lesions, particularly when compared with CT. Therefore, it might also be questionable what MRI may have to offer to outweigh the performance of PET/CT in lung cancer staging except for the lack of radiation exposure and superiority in staging brain metastases.

Preliminary results are available in recent literature on direct comparison between PET/MRI and PET/CT in the staging of lung cancer, mostly non-small-cell lung cancer (NSCLC) [17, 37-40]; however, many of these studies are limited by small sample size.

Schwenzer et al. [38] reported on ten patients with lung cancer and compared PET/CT with PET/MRI by applying a lesion-to-liver ratio to quantify FDG-avid lesions. They concluded there was similar lesion characterization and tumor detection between PET/CT and PET/MRI in most patients. The small sample size is certainly a major limitation of the study.

Similarly, PET/CT has been directly compared with whole-body MRI in 165 patients for the comprehensive staging of lung cancer with the conclusion that both PET/ $\mathrm{CT}$ and 3.0-T whole-body MRI appear to provide acceptable accuracy and comparable efficacy for NSCLC staging. However, for M-stage determination, each modality has its own advantages [39]. MRI was superior in detecting liver and brain metastases, whereas PET/CT performed better in the staging of lymphadenopathy [39]. The differences were not statistically significant.

The same authors also investigated the efficacy of coregistered PET/MRI and PET/CT images in correctly staging lung cancer in a preoperative setting with the aim of identifying correct upstaging, which would avoid unnecessary thoracotomy [41]. Although both staging tools allowed greater than $20 \%$ correct upstaging compared with conventional staging methods, co-registered PET/ MRI images did not appear to help identify significantly more correctly upstaged cancer patients than PET/CT plus brain MRI in patients with NSCLC.

Despite this statement, the capability of comprehensive assessment of both local and distant metastases including brain, liver, and bone [24, 26, 41] with high accuracy in a single imaging modality and examination may still be considered a significant advantage of PET/MRI over PET/ $\mathrm{CT}$ in addition to other aspects such as patient radiation exposure, patient comfort, and workflow management.

The performance of PET/MRI has also been studied for the detection of pulmonary nodules [28, 40, 42•]. With use of simultaneous PET and MRI acquisition and a freebreathing radial volumetric interpolated breath-hold examination, the detection of pulmonary nodules was compared with that in PET/CT. In 32 patients with a total of 69 nodules, including 45 FDG-avid lesions, the sensitivity of PET/MRI was $70.3 \%$ for all nodules, $95.6 \%$ for FDG-avid nodules, and $88.6 \%$ for nodules $0.5 \mathrm{~cm}$ in diameter or larger. PET/MRI had a higher sensitivity than PET for all nodules (70.3 vs $61.6 \%, P=0.002)$ and higher sensitivity than MRI alone for FDG-avid nodules (95.6 vs $80.0 \%, P=0.008$ ). There was a significantly strong correlation between $\mathrm{SUV}_{\max }$ of pulmonary nodules obtained with PET/CT and that obtained with PET/MRI $(r=0.96, P<0.001)$ [42•].

When a 3D Dixon-based, dual-echo gradient echo pulse sequence and a trimodality PET/CT/MRI setup was used in 40 patients, MRI detected 58 of 66 nodules seen on CT. Detection rates between CT and MRI were considered similar and not statistically significantly different $(P>0.05)$ [28].

What remains a challenge in the current stage of PET/ MRI, however, is the quantification of abnormal lesions in the PET component, which is based on MRAC. MRAC is still under investigation and is a work in progress. Different vendors favor different approaches. In general, attribution of $\mu$ values is dependent on the type of model being used for underlying tissue segmentation and attenuation map generation. All currently available segmentation approaches account for air, lung, and soft tissue but differ with respect to bone and fat segmentation. For the lung, it has been shown that, with use of a four-segment model, there is no statistically significant correlation between PET/MRI and PET/CT in the lung, subcutaneous fat, psoas muscle, and blood pool [43]. This variability in segmentation models still causes inconsistency and lacks credence in comparing system designs and techniques.

Our own research on nodal staging using a three-class segmentation algorithm focused on the quantitative assessment of SUVs in PET/MRI and PET/CT in the critical interface between lung tissue and mediastinal soft tissue. Since lung cancer lymphadenopathy is predictably located in this zone of greater risk of incorrect segmentation, quantification errors of lesions may occur.

Our results reflect experience similar to that previously reported. PET/MRI is feasible in patients with lung cancer and has reasonable accuracy regarding nodal stage [17]. Looking at the performance of PET/MRI in the nodal staging only, we were able to demonstrate that SUVs showed strong positive correlation (Spearman correlation of 0.93, $P<0.001$ ) between the two techniques with regard to FDG-avid lesions. Interobserver agreement in multiple observers was high $(\kappa=0.86)$ for PET/CT and substantial $(\kappa=0.70)$ for PET/MRI for the detection of lesions. The overall diagnostic performance of PET/MRI was slightly inferior to that of PET/CT, without statistical significance $(P>0.05)$ [17] (Fig. 1). 
Even given these promising preliminary results on the performance of PET/MRI in lung cancer, experience is still limited and based on small sample sizes. Furthermore, current knowledge is still sparse as to what quantification capacities PET/MRI has to offer in order to ascertain the reproducibility of SUVs and the reliability of SUV variations in follow-up examinations for comparison, specifically in these areas of critical segmentation. This means that the relevance of the current data remains to be determined, and further evidence is required to prove that PET/ MRI might have a benefit over current diagnostic algorithms in these patients with lung cancer.

\section{Pancreatic Cancer}

The generic term "pancreatic cancer" refers to a heterogeneous group of neoplasms, including pancreatic adenocarcinoma with or without mucinous features, neuroendocrine tumors, serous and mucinous cystic pancreatic neoplasms, intraductal papillary mucinous neoplasms, rare neoplasms such as solid papillary tumors of the pancreas, acinar and undifferentiated tumors, and nonepithelial tumors.

From an imaging perspective, these different neoplasms have very diverse imaging features in cross-sectional imaging and, more importantly, demonstrate a variable behavior in metabolic and functional imaging with PET. Hence the value of PET/CT is dependent on the individual underlying neoplasm.

For local staging of pancreatic cancer in general it is reported that multidetector-row $\mathrm{CT}$ and MRI/magnetic resonance cholangiopancreatography (MRCP) have comparable sensitivity and specificity rates for the diagnosis and staging of pancreatic cancers. Endoscopic ultrasonography offers the best sensitivity and specificity rates for lesions smaller than $2 \mathrm{~cm}$. Improved staging has been

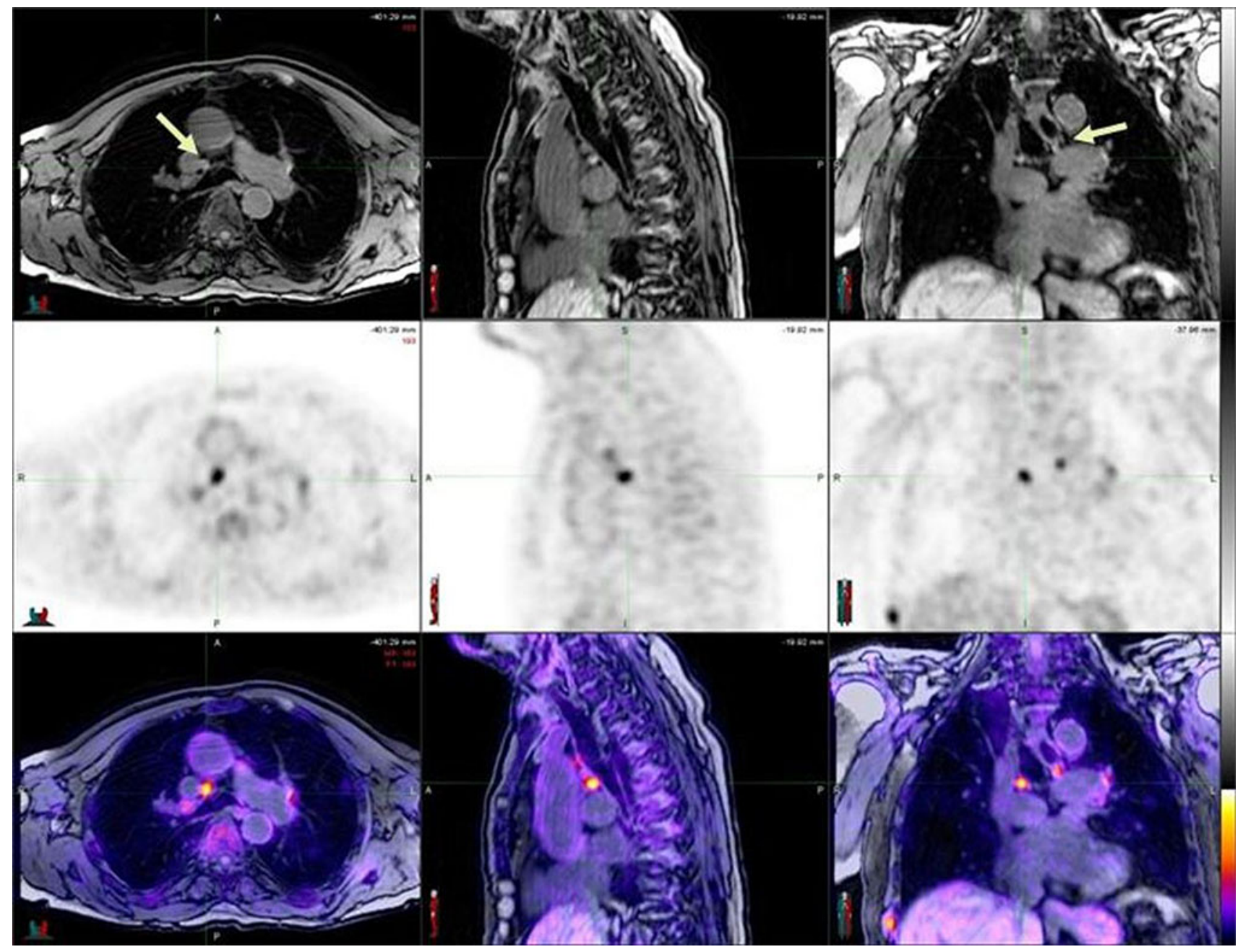

Fig. 1 Positron emission tomography (PET)/magnetic resonance imaging (MRI) in a patient with history of lung cancer. PET/MRI demonstrates two mediastinal lymph nodes up to $1 \mathrm{~cm}$ in size, which demonstrate 2-deoxy-2-( ${ }^{18}$ F)fluoro-D-glucose (FDG) avidity. Despite the small size, they are identified by both MRI and PET 
noted when PET/CT scans are added to preoperative evaluation [44].

In current clinical practice, contrast-enhanced $\mathrm{CT}$ is the modality of choice for diagnosis and staging of pancreatic cancer, irrespective of the underlying subtype. A "dual phase" technique after administration of iodinated contrast medium with image acquisition approximately $35-50 \mathrm{~s}$ and 60-70 s after the beginning of contrast medium injection yields a diagnostic accuracy of more than $90 \%$ for the detection of pancreatic cancer and positive predictive values for resectability of approximately $80 \%$ [45]. As such, contrast-enhanced CT can be considered the workhorse of pancreatic cancer staging.

The value of FDG-PET in pancreatic cancer is dependent on the type of primary neoplasm. Pancreatic adenocarcinoma typically expresses FDG avidity, and the diagnostic accuracy of FDG-PET for primary staging of pancreatic malignancy is reported to be up to $89 \%$ [46] with limitations in lymph node staging, yielding a negative predictive value of only $75 \%$ [47]. FDG-PET/CT is also reported to be specifically beneficial in the primary diagnosis of intraductal papillary mucinous neoplasms in distinguishing between benign and malignant neoplasms [48]. The greater value of FDG-PET, in general, however, is seen in the determination of metastatic disease and in the setting of treatment response monitoring and to a lesser degree, if at all, in the primary local staging. Even as such, the role of FDG-PET/CT still seems somewhat controversial given the fact that FDG avidity may be variable in pancreatic adenocarcinoma, with a significant overlap between benign and malignant lesions [49]. Neuroendocrine tumors generally exhibit low FDG avidity depending on the differentiation. In neuroendocrine tumors of the pancreas, somatostatin receptor binding PET ligands such as ${ }^{68} \mathrm{Ga}$-labeled octreotide have been demonstrated to be more efficient [50]. They are being used with great success in Europe and Asia; unfortunately, most of them are not FDA-approved and therefore are not clinically available in the USA.

Besides the limitations of FDG-PET/CT in pancreatic cancer as a single imaging modality, there is strong evidence in the literature that fusion of PET images with morphologic images and particularly MRI images significantly enhances accuracy in disease staging. For example, FDG-PET/MRI fusion significantly improved accuracy compared with that of PET/CT from 87 to $97 \%$ in a study on 119 patients [51]. A similar study on 47 patients confirmed this finding in that the diagnostic accuracy of PET/ T1-w MRI or PET/T2-w MRI was higher at 93 and $91 \%$, respectively, than that of PET/CT at $88 \%$, although statistical significance was not obtained [52]. Both sets of authors concluded that fused PET and MRI images were superior to PET/CT images in characterizing pancreatic tumors and distinguishing benign from malignant lesions.
Data on the staging of pancreatic cancer with dedicated PET/MRI or sequential PET and MRI are currently not available.

In our own experience covering 116 patients with oncologic disease, a small series of seven patients allowed us to gain a preliminary impression of what the potential of PET/MRI could be compared with individual imaging with PET/CT and MRI.

One of the most striking benefits of PET/MRI in pancreatic cancer in our opinion is its ability to provide comprehensive information about local $\mathrm{T}$ stage, resectability, and distant metastatic disease in a single examination (Fig. 2). From our preliminary experience on seven patients, PET/MRI improved the diagnostic confidence in TNM staging in four of seven patients over that of PET/CT, mainly in the T staging. In one patient, MRI was able to improve the confidence in the presence of hepatic metastases particularly owing to DWI, whereas the presence of metastatic disease was questionable in PET owing to poor FDG uptake and the small size of the metastatic lesions.

PET information in addition to CT and MRI information was distinctly helpful in three of seven patients who had postoperative changes after a Whipple procedure. While with morphologic imaging postoperative changes were difficult to distinguish from recurrent disease, PET added significant information in these cases and was able to rule out recurrence in one case. However, the soft tissue resolution of MRI was superior to that of low-dose CT to localize the site of recurrence in two cases within the postoperative changes.

For the quantification of FDG uptake, in our series of seven patients, 61 FDG-avid lesions were analyzed and compared between the modalities PET/CT and PET/MRI. $\mathrm{SUV}_{\text {max/mean }}$ in these lesions correlated strongly between the two modalities, with $R=0.84$ and $0.84(P<0.001)$, respectively.

Another clinical condition, which, according to our experience, will likely benefit from combined imaging with PET/MRI is the search for malignancy in the presence of chronic pancreatitis. Chronic pancreatitis can manifest itself as complex cystic, solid, and scirrhous destruction of the pancreatic parenchyma with calcifications. Solid tissue components with scar formation are indistinguishable from low-enhancing neoplasms, which is a well-known dilemma in pancreatic imaging with both MRI and CT.

Although distinguishing chronic or acute inflammatory disease from malignancy can also be challenging in PET owing to the overlap of SUVs of these two conditions, it can be expected that the direct image fusion of findings from both modalities will help improve the diagnostic confidence. Two of our patients benefited from this feature of PET/MRI, and $\mathrm{T}$ staging was facilitated given the excellent soft tissue contrast from MRI. 

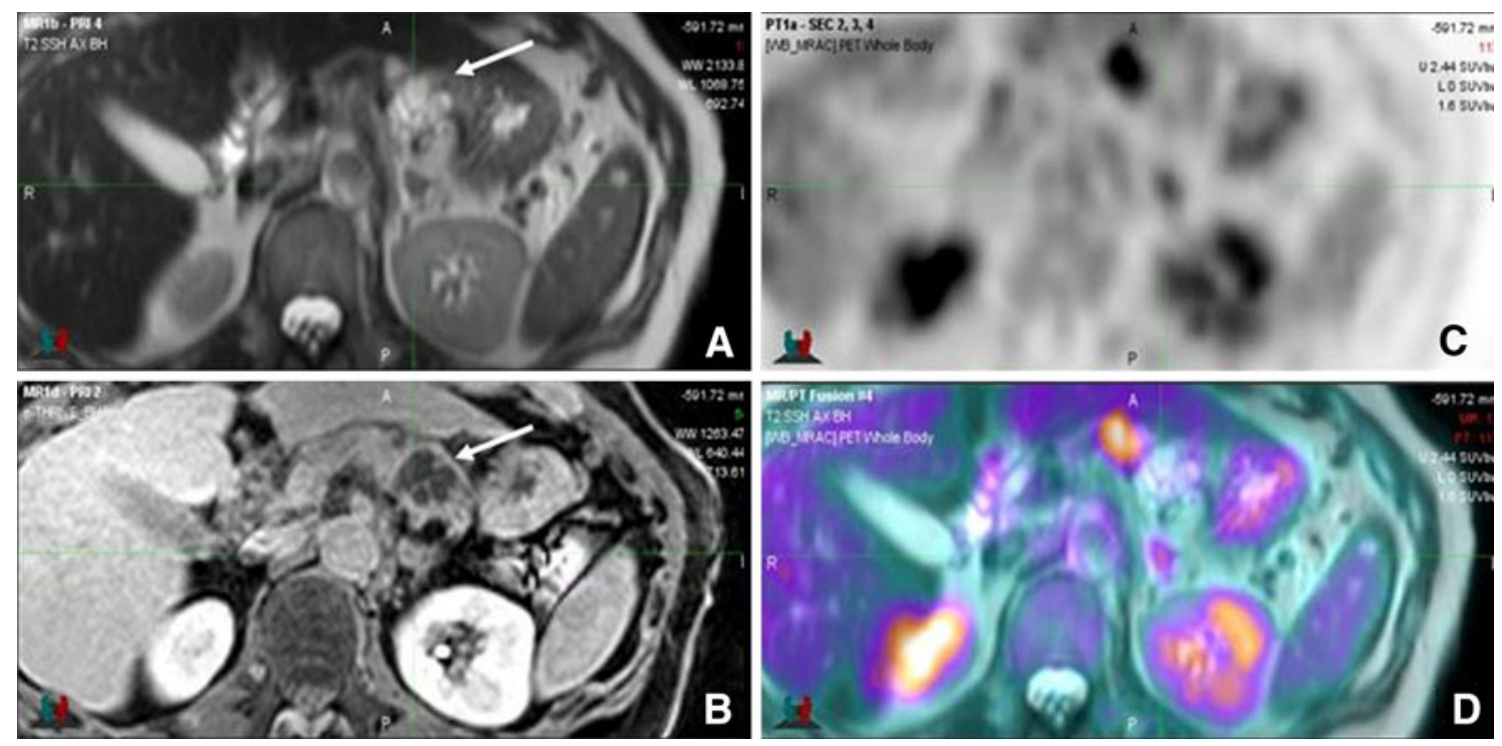

Fig. 2 PET/MRI in a 71-year-old woman presenting with a cystic mass in the pancreas which was suspected to be serous or mucinous neoplasm. PET/MRI shows increased FDG metabolism in the pancreatic body proximal to the cystic mass, which on fine-needle aspiration was confirmed to be pancreatic adenocarcinoma. MRI clearly demonstrates the exact extent of the cystic mass (arrow) and

Imaging protocols for pancreatic cancer assessment are straightforward. A dedicated pancreatic MRI protocol including the liver requires a total scanning time of approximately $30 \mathrm{~min}$ and can easily encompass, again, a whole-body mDIXON protocol and whole-body PET in a 45-min examination. Imaging protocols may be shorter when a simultaneous acquisition is available; however, most oncologic approaches as described for the simultaneous acquisition are also reported to revolve around an acquisition time of $30 \mathrm{~min}$, keeping in mind that simultaneous imaging is initiated after a tracer uptake time of $60 \mathrm{~min}$.

The joint value of PET and functional MRI for investigation of pancreatic cancer is a totally unexploited field in the current literature and experience with PET/MRI and studies addressing the combined value of DWI, DCE imaging, and PET in conjunction are to be welcomed in the future.

\section{Colorectal Cancer}

In colorectal cancer, both MRI and PET/CT are independently well established imaging modalities, and combining the two modalities into one will only strengthen their already high performance for this disease category. Again the expected benefit in this neoplasm is the hope for a onestop-shop modality for comprehensive TNM staging with a single imaging examination.

the enhanced nodule proximal to it, which is limited to the pancreatic body and represents the actual cancer. Thus, MRI provides the local tumor staging, and both PET and MRI provide the staging of distant metastatic disease, opening up the possibility for PET/MRI to become a one-stop-shop imaging modality for the primary staging of this disease

MRI has evolved to a key imaging modality in the local staging of rectal cancer and in the assessment of metastatic disease of the liver. In both instances, MRI has proven to be superior to CT and even PET/CT [53].

FDG-PET/CT is generally established in the staging of metastatic colorectal cancer and the assessment of disease recurrence after therapy [54]; however, it has demonstrated some limitations if used as a single modality.

To evaluate nodal stage in colorectal cancer, the current literature does not recommend the routine use of FDG$\mathrm{PET} / \mathrm{CT}$ as a single staging tool [55]. PET/CT appears to be limited in smaller nodes, owing to limited spatial resolution and uptake quantity.

FDG-PET/CT has the ability to accurately predict the presence and size of liver metastases; however, this depends on the FDG avidity of the tumor and its metastases and the degree of differentiation of the primary tumor. FDG-PET/CT is of questionable value in the setting after chemotherapy, where fatty changes in the liver hamper the achievement of appropriate accuracy. In these conditions, $\mathrm{PET} / \mathrm{CT}$ appears to be inferior to contrast-enhanced CT in the detection of disease [56].

In both of these instances, MRI is of distinct value to complement PET/CT owing to its high soft tissue contrast and strengths in lesion detection, particularly when DWI is used.

Dedicated PET/MRI data in colorectal cancer are currently not available. Preliminary information has been 
reported on various oncologic diseases in patients, including colorectal cancer patients without distinct focus on colorectal cancer [57].

A distinct benefit may arise from the combined use of MRI and PET/CT to detect lymph node involvement in rectal cancer. The high soft tissue contrast and morphologic criteria established in MRI for assessment of metastatic nodal involvement may complement the lack of sensitivity of PET/CT alone in this application (Fig. 3).

A typical imaging protocol for colorectal cancer staging and workup with PET/MRI with the sequential scanner approach would ideally include the local staging for rectal cancer (if applicable) in the pelvis and at the same time assessment for liver metastases. Our protocol has the following design: $\mathrm{T} 2 \mathrm{w}$ single-shot imaging in two planes for the liver, abdomen, and pelvis, T2w single-shot imaging with fat saturation for the liver and DWI for the liver and pelvis in an axial orientation, and $\mathrm{T} 2 \mathrm{w}$ fast spin echo (turbo spin echo) imaging in three planes for the pelvis and rectum if the primary cancer is rectal cancer. Preconstrast and postcontrast images are acquired for the liver, including dynamic scanning $25 \mathrm{~s}, 70 \mathrm{~s}$, and $3 \mathrm{~min}$ after injection of the contrast medium and precontrast and postcontrast imaging of the pelvis in two planes. A whole-body mDIXON sequence is then applied for wholebody anatomic correlation of distant metastatic foci. The mDIXON sequence is applied both before and after injection of the contrast medium, and adds only a few minutes to the total protocol. Typically, such combined morphologic and whole-body protocols can be performed in 35-40 min depending on the body size of the patient. In a sequential approach, the PET component adds another approximately $15 \mathrm{~min}$ to the MRI examination time; however, most of the examination can be performed within $1 \mathrm{~h}$.

In summary, the potential benefits of PET/MRI may lie in the combination of the two modalities since they express complementary strengths and weaknesses. From our current limited experience we can speculate that $\mathrm{N}$ staging will benefit from the added improved morphologic assessment of lymph nodes beyond size in adding MRI. Liver metastatic disease will improve with better detection in MRI and even in MRI with liver-specific agents. Both modalities have the potential of improving overall TNM staging in colorectal cancer. Including functional MRI with DWI and possibly DCE imaging in the evaluation and combining these functional parameters with the molecular information provided by PET tracers has not been explored in the current literature to date. This additional aspect opens up further potential for improvement to outperform the current performance of PET/CT only as a single diagnostic modality.
Ovarian Cancer

Primary diagnosis of ovarian cancer is established as a combination of information from clinical examination, ultrasound studies, levels of serum markers such as CA125 , CT, and eventually postoperative histopathology. The goal of imaging in ovarian cancer detection is to expeditiously distinguish benign adnexal lesions from those requiring further pathologic evaluation for malignancy.

For primary diagnosis of ovarian cancer, MRI has a limited role, although its performance is excellent. For lesions indeterminate on ultrasonography, MRI increases the specificity of imaging evaluation, thus decreasing unnecessary resections of benign masses. MRI is reported to have a sensitivity and specificity of $100 \%$ and $94 \%$, respectively, in the evaluation of sonographically indeterminate ovarian masses [58]. However, owing to its limited availability and relatively high cost, MRI is more often used as a problem-solving tool in the case of indeterminate lesions.

FDG-PET/CT has shown expanding use for treatment planning and follow-up in ovarian cancer; however, it is not recommended as the modality of choice for primary detection of cancer. False-negative results in borderline indeterminate ovarian neoplasms have occurred; and falsepositive results may be generated when benign lesions such as endometriosis and leiomyomata and also normal follicles in premenopausal women exhibit increased FDG activity and are mistaken as cancer. On the other hand, increased FDG uptake in the ovary in postmenopausal women is considered abnormal and suggestive of malignancy. CT is useful in diagnosis and treatment planning of advanced ovarian cancer.

In the assessment of recurrent ovarian cancer, both PET/ CT and MRI are strong imaging modalities with high sensitivity and specificity in detecting tumor recurrence. Interestingly, there is literature which has demonstrated superiority of PET/CT over MRI and vice versa.

The sensitivity, specificity, positive predictive value, negative predictive value, and diagnostic accuracy of PET/ CT were reported to be $97.5,100,100,87.5$, and $97.8 \%$, respectively, whereas those of MRI were 95, 85.7, 97.4, 75, and $93.6 \%$, respectively. For smaller peritoneal implants less than $2 \mathrm{~cm}$ and less than $1 \mathrm{~cm}$ in size, the sensitivity, negative predictive value, and diagnostic accuracy of PET/ CT were significantly better than those of MRI $(P<0.05)$ [59]. In the setting of rising levels of tumor markers after remission, PET/CT is recommended as the modality of choice for detection of tumor recurrence.

Despite its high performance in detection of recurrent disease, FDG-PET/CT has a number of limitations. PET/ CT may yield false-negative results in patients with small, 


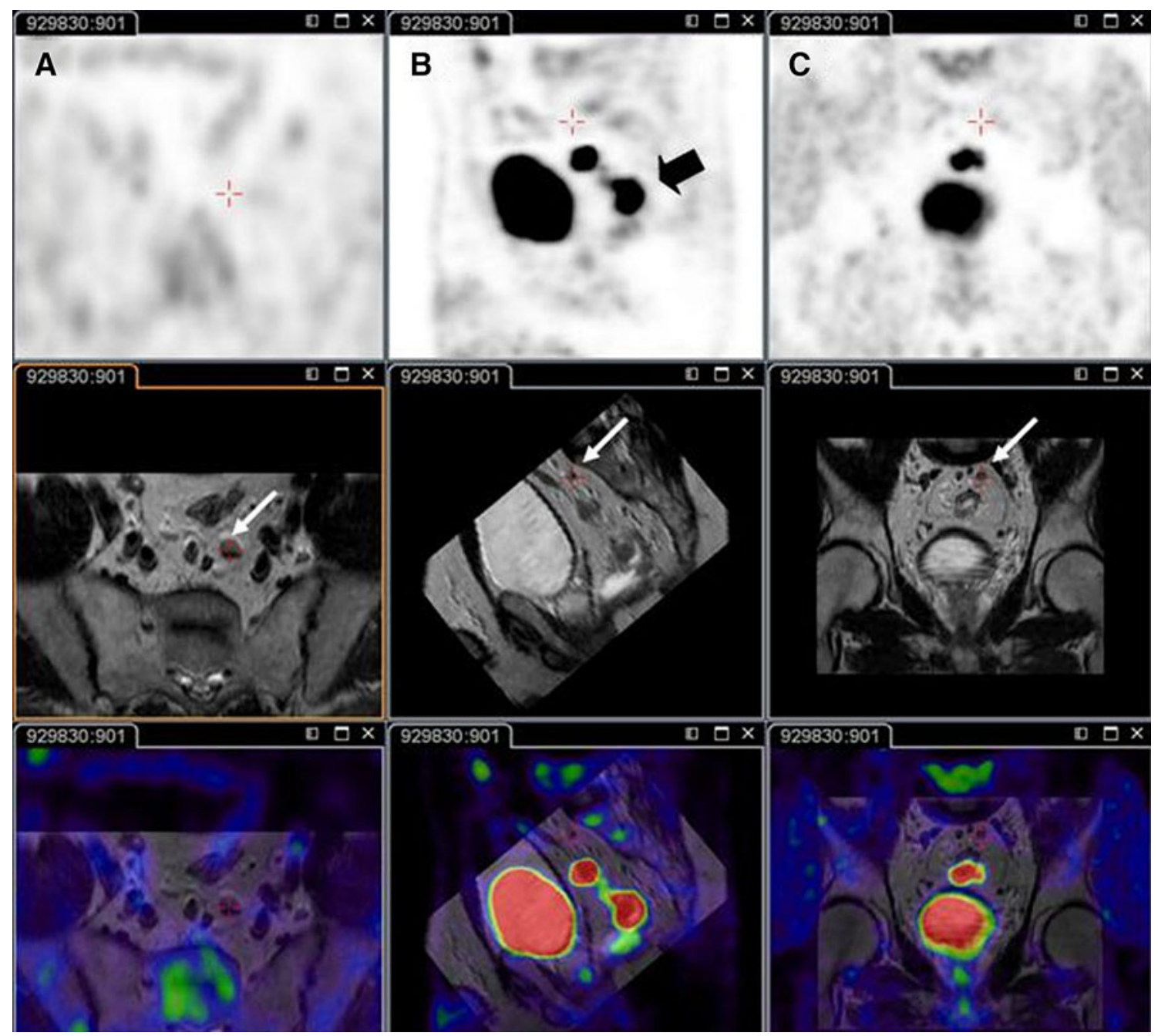

Fig. 3 PET/MRI in a patient with rectal cancer. Axial (column $A$ ), sagittal (column $B$ ), and coronal (column $C$ ) PET/MRI images of the pelvis. The primary tumor demonstrates clearly abnormally increased FDG uptake (column $B$, large arrow). A pelvic lymph node is seen on

necrotic, mucinous, cystic, or low-grade tumors. In the posttherapy setting, false positives can arise from inflammatory and infectious processes and may lead to misinterpretation. In addition, normal intense bladder and ureteral excreted activity in FDG-PET/CT can obscure disease in the pelvis. Minimizing these artifacts is important in protocol design in both PET/CT and PET/MRI.

The value and high effectiveness of MRI in ovarian cancer recurrence was demonstrated as early as in 2000 and has been confirmed several times since then [60]. Kim et al. [61] reported patient-based sensitivity and accuracy of PET/CT and MRI for detection of recurrent ovarian cancer of 73 and $91 \%(P<0.05)$ and 81 and $89 \%(P>0.05)$, respectively. The overall lesion-based sensitivity of PET/ CT and MRI was 66 and $86 \%$, respectively $(P<0.05)$. In detecting peritoneal lesions, the overall lesion-based sensitivity and accuracy of PET/CT and MRI for peritoneal
MRI, which on MRI is enlarged and demonstrates criteria of metastatic involvement. This lymph node does not demonstrate increased FDG avidity

lesions were 43 and $86 \%$ and 75 and $94 \%$, respectively $(P<0.05)$. Kim et al. [61] concluded that MRI is superior to PET/CT in detecting disease recurrence.

In this field of slightly contradictory literature, PET/ MRI seems to dissolve the competition between the two modalities and unifies the strengths of both in one. Data on the performance of PET/MRI in ovarian cancer in both primary diagnosis and recurrence are not currently available.

In our experience, the two modalities are distinctly complementary and synergistic. Since the FDG avidity of the tumor is indeed dependent on the cellularity and mucinous content, MRI may ideally compensate for this limitation of PET. DWI is distinctively helpful in the detection of lymphadenopathy, peritoneal implants, and local recurrence, even if these are small and irrespective of the mucinous content. In contrast, MRI may be limited in 
identifying small serosal implants on the surface of the small bowel if the implants are soft tissue in nature. Although bowel implants may be mistaken as bowel activity in PET/CT, the combined approach with high soft tissue resolution and abnormal FDG uptake helps to even out limitations on both sides. Especially in the subdiaphragmatic area, where PET/CT can be subject to image quality impairment and misregistration from respiratory motion, the breath-hold imaging capacity of MRI is of particular value to improve accuracy and diagnostic confidence (Fig. 4).

With both modalities superimposed, our experience shows that the subjective diagnostic confidence regarding the presence or absence of disease increases to a substantial degree.

\section{Lymphoma}

The National Comprehensive Cancer Network of the USA has recently released new guidelines on the diagnosis, treatment, and follow-up of lymphoma and has established the Deauville criteria in PET for treatment response assessment, replacing the International Working Group response criteria [62]. PET and integrated PET/CT are increasingly being used to assess treatment response of lymphoma during therapy. Interim PET scans are being performed for this purpose. There is evidence in the literature that early interim PET after chemotherapy is a sensitive prognostic indicator of treatment outcome in patients with advanced disease (stage II disease with unfavorable risk factors or stage III-IV disease) [62]. Interim PET after two or three cycles of chemotherapy may predict progression-free survival and overall survival and could be considered in the prognostic evaluation of diffuse large B cell lymphoma and similarly for follicular lymphoma and primary cutaneous lymphoma [63, 64]. However, the diagnostic and prognostic value has not been definitely established for early disease in lymphoma [62].

With the recommendation of frequent PET/CT however, there is significant accumulation of radiation over time, which raises concerns specifically in young patient populations [65]. The expected benefit of PET/MRI in this regard appears obvious in that radiation exposure can be limited to the dose generated by the radioactive tracer. Significant reduction of the cumulative radiation dose to the patient can be achieved over time when additional CT radiation exposure is avoided. Hence, the performance of PET/MRI is of particular interest for patients with lymphoma and their physicians in view of its potential to reduce the radiation burden.

The second major interesting topic is whether PET/MRI including its combined functional (DWI), morphologic, and metabolic (FDG) components will be capable of changing the regimens and algorithms of treatment followup with PET in lymphoma or will add an alternative to the current diagnostic imaging algorithms. Distinct value has been attributed to DWI in lymphoma. DWI and apparent diffusion coefficient (ADC) quantification correlates well with PET avidity in PET/CT [66] and has predictive capacity for treatment response. At the same time, PET has been advocated to guide clinical biopsies and risk-adapted therapy. Direct correlation between ADC and PET avidity and their joint incremental benefit will be a fascinating aspect of PET/MRI to clarify.

Initial results of direct comparison between PET/MRI and PET/CT for lesion detection and localization in lymphoma promise high overall concordance. In the first clinical experience with integrated whole-body PET/MRI (Biograph mMR; Siemens Healthcare) in oncologic disease, Drzezga et al. [57] evaluated the combined studies of PET/CT and PET/MRI of 32 patients, two of whom presented with the diagnosis of lymphoma. According Drzezga et al. [57], corresponding PET/CT and PET/MRI images did not show a significant difference regarding the numbers of suspicious lesions or positive lesions, with a comparable anatomic allocation.

Another recent retrospective study [67•] assessing the feasibility of FDG-PET/MRI for response evaluation of malignant lymphoma looked at nine patients with malignant lymphoma. All patients underwent FDG-PET/MRI twice, once for staging and once for therapy response assessment. In two patients, both the initial examination and the follow-up examination included additional diffusion-weighted MRI (DWI). Two nuclear medicine physicians, who evaluated independently the PET scans, reported high interobserver agreement in staging the disease. In addition, all PET data sets were found to have very good or excellent image quality. The additional DWI performed in two patients confirmed its technical feasibility. However, the authors of the study concluded that its clinical role has still to be defined [67•].

Our own experience with lymphoma in PET/MRI is very consistent with the reported results in the literature. In our retrospective multireader analysis of ten patients with malignant lymphoma who underwent FDG-PET/CT followed by PET/MRI (Philips Ingenuity TF PET/MR, Philips Healthcare, Andover, MA, USA) in one imaging session, a high correlation of findings in terms of localization and characterization was found. Excellent intraobserver agreement was found regarding the number of lymphomatous lesions detected with PET/CT and PET/MRI, with an equal-weighted $\kappa$ of $\kappa_{1}=0.84$ and $\kappa_{2}=0.86$, respectively. Interreader agreement was slightly lower $(\kappa=0.4)$ for the grading of the lesions (reader 1 rated more lesions in PET/MRI as "pathologic," whereas reader 2 rated those as "suspicious") but not for the presence of the disease. 


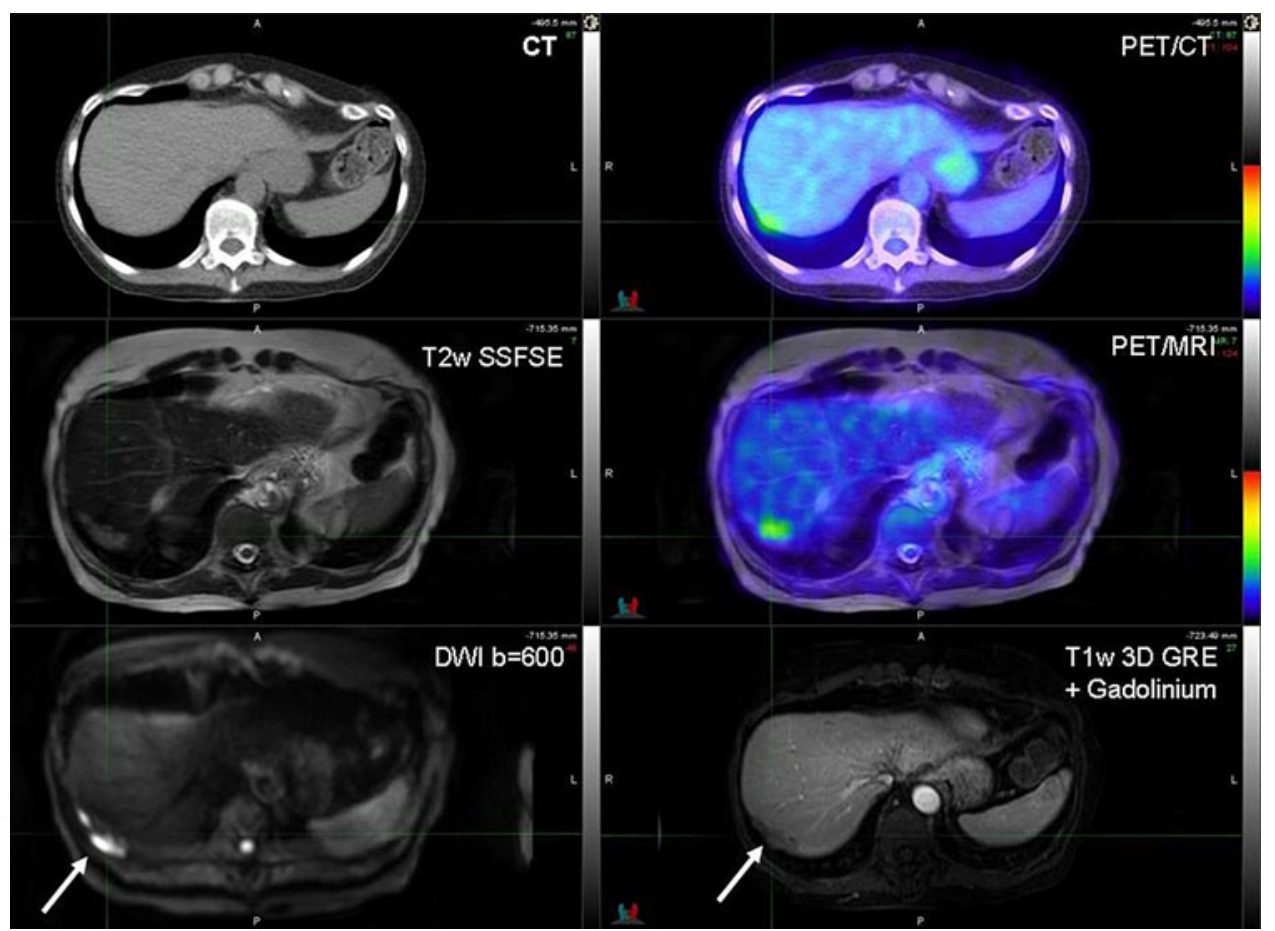

Fig. 4 PET/MRI in a female patient with a history of ovarian cancer: PET/computed tomography $(C T)$ identified focally increased tracer uptake in the area close to segment 7 of the liver; however, a lesion is barely visualized on nonenhanced low-dose CT. No clear distinction could be made whether this lesion is located in the lower lung base, in the pleura, within the liver, or in the subdiaphragmatic space on PET/ CT. MRI demonstrates a moderately enhancing mass [T1-weighted

Lesion localization was congruent between corresponding PET/MRI and PET/CT for reader 2, resulting in the same stage of the disease according to the Ann Arbor classification. Reader 1 detected one additional bone lesion on PET/MRI, which was not visible on PET/CT. This finding changed the stage of the disease and is an excellent example of the superiority of MRI in the detection of bone marrow involvement in lymphomatous disease.

The imaging protocol for lymphoma patients is less complex than for other clinical indications, and ideally includes whole-body imaging with $\mathrm{T} 2 \mathrm{w}$ fat-saturated and T1w imaging in order to assess the patient for lymphadenopathy and potential bony involvement. Whole-body short inversion time inversion recovery half-Fourier rapid acquisition with relaxation enhancement MRI has been advocated with high accuracy for the same indication [68]. Whole-body DWI should be added for better lymph node detection and ADC quantification in order to evaluate therapy response. MRI may be acquired in axial planes; however, coronal acquisition has advantages in terms of timing. Overall, a comprehensive whole-body protocol in MRI is feasible in $20 \mathrm{~min}$, additive to the PET component of approximately $15 \mathrm{~min}$. Contrast medium application
(T1w) gradient echo (GRE) imaging; small arrow] which is clearly located in the subdiaphragmatic space, indenting the liver parenchyma of segment 7 , and can be identified as a peritoneal implant of the ovarian cancer. Note the high conspicuity of the lesion on diffusion-weighted imaging ( $D W I$; small arrow) compared to SSFSE single-shot fast spin echo imaging

may be optional since with appropriate nonenhanced imaging sequences, differentiation between lymph nodes and vessels should not pose a problem.

More critical than lesion detection is the change of metabolic activity of lymphomatous lesions both initially and in treatment response evaluation. Currently, visual analysis based on liver uptake or mediastinal blood pool is used for follow-up imaging with PET (Deauville criteria) [69]. Although these measures are well accepted for PET/ CT, they have not been evaluated for PET/MRI. Furthermore, the consistency and reproducibility of regional FDG uptake in PET/MRI has not been tested to a sufficient degree to allow them to be of comparable diagnostic confidence. Again, the robustness of segmentation and accurate attenuation correction are essential prerequisites to provide this quantification in PET/MRI, and further studies are warranted to prove evidence of its diagnostic confidence.

\section{Pediatric Neoplasms}

The value of combining PET and MRI has been demonstrated extensively in the world of pediatric oncology, not 
only for neuroradiologic applications but also in children with sarcoma, lymphoma, small cell cancers, and other cancers which also benefit from the physiologic characterization and metabolic information from PET [68, 7073].

For pediatric patients, the special benefits of PET/MRI compared with PET/CT in one examination obviously lie again in the reduced radiation exposure when substituting the CT component with MRI, and in the expected improvement of the logistics and workflow of such complex examinations.

Reports on initial experience with PET/MRI in children have proven its feasibility, with a technical success rate of $100 \%$. Hirsch et al. [74•] state that PET/MRI saves time and resources. Within the limits of small patient populations of 15 patients, the results are encouraging, demonstrating the synergistic character of the two imaging modalities. Although FDG-PET detected ten more metabolically active lymph node areas, MRI identified three more sites of organ involvement in lymphoma [74•]. Of interest is hereby disease involvement detected by MRI of organs in which PET typically demonstrated physiologic uptake such as the kidneys and the liver, a merit of high soft tissue capacities in MRI.

\section{Conclusion and Future Perspectives}

Current evidence shows overall good correlation between $\mathrm{PET} / \mathrm{CT}$ and PET/MRI in lesion detection across most publications and oncologic diseases. However, there is lack of clearly defined prospective studies with a sufficient number of patients to validate these findings.

Initial results in pediatric patients are encouraging, particularly with the reduction in radiation exposure. However, there is not yet an established indication for which PET/CT can be replaced by PET/MRI. For some tumors, a dedicated benefit of PET/MRI is represented in the potential for a combined one-stop-shop modality and in the comprehensive TNM staging plus potentially improved $\mathrm{N}$ staging in the abdomen (i.e., pancreatic, colorectal, and gynecologic cancers).

Defined PET/MRI protocols need to be developed and validated which go beyond adding two imaging modalities. If the question is lesion characterization and either PET or MRI has already sufficiently answered the question, the full range of MRI sequences applied to dedicated organ protocols might not be necessary.

Further improvements in the MRAC are essential to provide accurate quantification of regional PET tracer uptake, which is important for treatment monitoring, specifically for early prediction of treatment response. An important potential future of PET/MRI might be in the use of non-FDG tracers, which might allow monitoring of individualized treatment decisions. A major task will be to explore and determine the value of combined functional, quantitative, and morphologic imaging in MRI with simultaneous molecular and metabolic information from PET.

\section{Compliance with Ethics Guidelines}

Conflict of Interest N. Avril's institution is funded by a grant from Philips Healthcare. A. A. Kohan, C. Rubbert, J. L. Vercher-Conejero, P. F. Faulhaber, and P. R. Ros are all funded as part of a research agreement between Philips Healthcare and Case Western Reserve University. A. Kalemis is an employee of Philips Healthcare. K. A. Herrmann, M. C. Gaeta, R. M. Paspulati, and B. Mansoori declare that they have no conflict of interest.

Human and Animal Rights and Informed Consent This article does not contain any studies with human or animal subjects performed by any of the authors.

\section{References}

Papers of particular interest, published recently, have been highlighted as:

- Of importance

1. Pichler BJ, Wehrl HF, Kolb A, Judenhofer MS. Positron emission tomography/magnetic resonance imaging: the next generation of multimodality imaging? Semin Nucl Med. 2008;38(3):199-208.

2. Schlemmer HP, Pichler BJ, Schmand M, et al. Simultaneous MR/ PET imaging of the human brain: feasibility study. Radiology. 2008;248(3):1028-35.

3. Delso G, Fürst S, Jakoby B, et al. Performance measurements of the Siemens mMR integrated whole-body PET/MR scanner. J Nucl Med. 2011;52(12):1914-22.

4. - Quick HH, von Gall C, Zeilinger M, et al. Integrated wholebody PET/MR hybrid imaging: clinical experience. Invest Radiol. 2013;48(5):280-9. doi:10.1097/RLI.0b013e3182845a08. This work is one of the first articles to report on experience with PET/ $M R I$ in a large patient population. It offers an initial idea of the performance of PET/MRI compared with PET/CT.

5. Kalemis A, Delattre BM, Heinzer S. Sequential whole-body PET/ MR scanner: concept, clinical use, and optimisation after two years in the clinic. The manufacturer's perspective. MAGMA. 2013;26(1):5-23.

6. Kuhn FP, Crook DW, Mader CE, et al. Discrimination and anatomical mapping of PET-positive lesions: comparison of CT attenuation-corrected PET images with coregistered MR and CT images in the abdomen. Eur J Nucl Med Mol Imaging. 2013;40(1):44-51.

7. von Schulthess GK, Kuhn FP, Kaufmann P, Veit-Haibach P. Clinical positron emission tomography/magnetic resonance imaging applications. Semin Nucl Med. 2013;43(1):3-10.

8. Veit-Haibach P, Kuhn FP, Wiesinger F, et al. PET-MR imaging using a tri-modality PET/CT-MR system with a dedicated shuttle in clinical routine. MAGMA. 2013;26(1):25-35.

9. Budinger TF. Time-of-flight positron emission tomography: status relative to conventional PET. J Nucl Med. 1983;24(1):73-8.

10. Murray I, Kalemis A, Glennon J, et al. Time-of-flight PET/CT using low-activity protocols: potential implications for cancer 
therapy monitoring. Eur J Nucl Med Mol Imaging. 2010;37(9):1643-53.

11. El Fakhri G, Surti S, Trott CM, Scheuermann J, Karp JS. Improvement in lesion detection with whole-body oncologic time-of-flight PET. J Nucl Med. 2011;52(3):347-53.

12. Martinez-Moller A, Souvatzoglou M, Delso G, Bundschuh RA, Chefd'hotel C, Ziegler SI, Navab N, Schwaiger M, Nekolla SG. Tissue classification as a potential approach for attenuation correction in whole-body PET/MRI: evaluation with PET/CT data. J Nucl Med. 2009;50(4):520-6.

13. Schulz V, Torres-Espallardo I, Renisch S, Hu Z, Ojha N, Bornert P, Perkuhn M, Niendorf T, Schafer WM, Brockmann H, Krohn T, Buhl A, Gunther RW, Mottaghy FM, Krombach GA. Automatic, three-segment, MR-based attenuation correction for whole-body PET/MRI data. Eur J Nucl Med Mol Imaging. 2011;38(1): 138-52.

14. Partovi S, Kohan A, Gaeta C, Rubbert C, Vercher-Conejero JL, Jones RS, O'Donnell JK, Wojtylak P, Faulhaber P. Image quality assessment of automatic three-segment MR attenuation correction vs. CT attenuation correction. Am J Nucl Med Mol Imaging. 2013;3(3):291-9.

15. Kershah S, Partovi S, Traughber BJ, Muzic RF Jr, Schluchter MD, O'Donnell JK, Faulhaber P. Comparison of standardized uptake values in normal structures between PET/CT and PET/ MRI in an oncology patient population. Mol Imaging Biol. 2013. doi:10.1007/s11307-013-0629-8.

16. Eiber M, Martinez-Möller A, Souvatzoglou M, Holzapfel K, Pickhard A, Löffelbein D, Santi I, Rummeny EJ, Ziegler S, Schwaiger M, ekolla SG, Beer AJ. Value of Dixon based MRPET attenuation correction sequence for the localization and evaluation of PET-positive lesions. Eur J Nucl Med Mol Imaging. 2011;38:1691-701.

17. Kohan AA, Kolthammer JA, Vercher-Conejero JL, Rubbert C, Partovi S, Jones R, Herrmann KA, Faulhaber P. N staging of lung cancer patients with PET/MRI using a three-segment model attenuation correction algorithm: Initial experience. Eur Radiol. 2013. doi:10.1007/s00330-013-2914-y.

18. Kim JH, Lee JS, Song IC, Lee DS. Comparison of segmentationbased attenuation correction methods for PET/MRI: evaluation of bone and liver standardized uptake value with oncologic PET/CT data. J Nucl Med. 2012;53(12):1878-82.

19. Samarin A, Burger C, Wollenweber SD, Crook DW, Burger IA, Schmid DT, von Schulthess GK, Kuhn FP. PET/MR imaging of bone lesions-implications for PET quantification from imperfect attenuation correction. Eur J Nucl Med Mol Imaging. 2012; 39:1154-60.

20. - Martinez-Möller A, Eiber M, Nekolla SG, Souvatzoglou M, Drzezga A, Ziegler S, Rummeny EJ, Schwaiger M, Beer AJ. Workflow and scan protocol considerations for integrated wholebody PET/MRI in oncology. J Nucl Med. 2012;53(9):1415-26. Workflow is an important issue in PET/MRI and is dependent on the clinical request, the desire for detail in the diagnosis, and the design of the imaging device. This article provides some valuable suggestions for the design of imaging protocols.

21. Platzek I, Beuthien-Baumann B, Langner J, Popp M, Schramm G, Ordemann R, Laniado M, Kotzerke J, van den Hoff J. PET/MR for therapy response evaluation in malignant lymphoma: initial experience. MAGMA. 2013;26(1):49-55.

22. Platzek I, Beuthien-Baumann B, Schneider M, Gudziol V, Langner J, Schramm G, Laniado M, Kotzerke J, van den Hoff J. PET/MRI in head and neck cancer: initial experience. Eur J Nucl Med Mol Imaging. 2013;40(1):6-11.

23. Vargas MI, Becker M, Garibotto V, Heinzer S, Loubeyre P, Gariani J, Lovblad K, Vallee JP, Ratib O. Approaches for the optimization of MR protocols in clinical hybrid PET/MRI studies. MAGMA. 2013;26(1):57-69.
24. Schmidt GP, Baur-Melnyk A, Haug A, Utzschneider S, Becker CR, Tiling R, Reiser MF, Hermann KA. Whole-body MRI at 1.5 $\mathrm{T}$ and $3 \mathrm{~T}$ compared with FDG-PET-CT for the detection of tumour recurrence in patients with colorectal cancer. Eur Radiol. 2009;19(6):1366-78.

25. Lauenstein TC, Goehde SC, Herborn CU, Goyen M, Oberhoff C, Debatin JF, Ruehm SG, Barkhausen J. Whole-body MR imaging: evaluation of patients for metastases. Radiology. 2004;233(1): 139-48.

26. Schmidt GP, Wintersperger B, Graser A, Baur-Melnyk A, Reiser MF, Schoenberg SO. High-resolution whole-body magnetic resonance imaging applications at 1.5 and 3 Tesla: a comparative study. Invest Radiol. 2007;42(6):449-59.

27. Sadick M, Schoenberg SO, Hoermann K, Sadick H. Current oncologic concepts and emerging techniques for imaging of head and neck squamous cell cancer. GMS Curr Top Otorhinolaryngol Head Neck Surg. 2012;11:Doc08.

28. Stolzmann P, Veit-Haibach P, Chuck N, Rossi C, Frauenfelder T, Alkadhi H, von Schulthess G, Boss A. Detection rate, location, and size of pulmonary nodules in trimodality PET/CT-MR: comparison of low-dose CT and Dixon-based MR imaging. Invest Radiol. 2013;48(5):241-6.

29. Kresnik E, Mikosch P, Gallowitsch HJ, Kogler D, Wiesser S, Heinisch M, Unterweger O, Raunik W, Kumnig G, Gomez I, Grünbacher G, Lind P. Evaluation of head and neck cancer with 18F-FDG PET: a comparison with conventional methods. Eur J Nucl Med. 2001;28(7):816-21.

30. Dresel S, Grammerstorff J, Schwenzer K, Brinkbäumer K, Schmid R, Pfluger T, Hahn K. [18F]FDG imaging of head and neck tumours: comparison of hybrid PET and morphological methods. Eur J Nucl Med Mol Imaging. 2003;30(7):995-1003.

31. Kim SY, Kim JS, Yi JS, Lee JH, Choi SH, Nam SY, Cho KJ, Lee SW, Kim SB, Roh JL. Evaluation of 18F-FDG PET/CT and CT/ MRI with histopathologic correlation in patients undergoing salvage surgery for head and neck squamous cell carcinoma. Ann Surg Oncol. 2011;18(9):2579-84.

32. Nakamoto Y, Tamai K. Clinical value of image fusion from MR and PET in patients with hand and neck cancer. Mol Imaging Biol. 2009;11:46-51.

33. Berker Y, Franke J, Salomon A, Palmowski M, Donker HC, Temur Y, Mottaghy FM, Kuhl C, Izquierdo-Garcia D, Fayad ZA, Kiessling F, Schulz V. MRI-based attenuation correction for hybrid PET/MRI systems: a 4-class tissue segmentation technique using a combined ultrashort-echo-time/Dixon MRI sequence. J Nucl Med. 2012;53(5):796-804.

34. Eiber M, Souvatzoglou M, Pickhard A, Loeffelbein DJ, Knopf A, Holzapfel K, Martinez-Möller A, Nekolla SG, Scherer EQ, Schwaiger M, Rummeny EJ, Beer AJ. Simulation of a MR-PET protocol for staging of head-and-neck cancer including Dixon MR for attenuation correction. Eur J Radiol. 2012;81(10): 2658-65.

35. Hatakenaka M, Nakamura K, Yabuuchi H, Shioyama Y, Matsuo Y, Ohnishi K, Sunami S, Kamitani T, Setoguchi T, Yoshiura T, Nakashima T, Nishikawa K, Honda H. Pretreatment apparent diffusion coefficient of the primary lesion correlates with local failure in head-and-neck cancer treated with chemoradiotherapy or radiotherapy. Int J Radiat Oncol Biol Phys. 2011;81(2): 339-45.

36. King AD, Chow KK, Yu KH, Mo FK, Yeung DK, Yuan J, Bhatia KS, Vlantis AC, Ahuja AT. Head and neck squamous cell carcinoma: diagnostic performance of diffusion-weighted MR imaging for the prediction of treatment response. Radiology. 2013;266(2): $531-8$.

37. Li J, Xu W, Kong F, Sun X, Zuo X. Meta-analysis: accuracy of 18FDG PET-CT for distant metastasis staging in lung cancer patients. Surg Oncol. 2013. doi:10.1016/j.suronc.2013.04.001. 
38. Schwenzer NF, Schraml C, Müller M, Brendle C, Sauter A, Spengler W, Pfannenberg AC, Claussen CD, Schmidt H. Pulmonary lesion assessment: comparison of whole-body hybrid MR/PET and PET/CT imaging-pilot study. Radiology. 2012;264(2):551-8.

39. Yi CA, Shin KM, Lee KS, Kim BT, Kim H, Kwon OJ, Choi JY, Chung MJ. Non-small cell lung cancer staging: efficacy comparison of integrated PET/CT versus 3.0-T whole-body MR imaging. Radiology. 2008;248(2):632-42.

40. Schmidt H, Brendle C, Schraml C, Martirosian P, Bezrukov I, Hetzel J, Müller M, Sauter A, Claussen CD, Pfannenberg C, Schwenzer NF. Correlation of simultaneously acquired diffusionweighted imaging and 2-deoxy-[18F] fluoro-2-D-glucose positron emission tomography of pulmonary lesions in a dedicated wholebody magnetic resonance/positron emission tomography system. Invest Radiol. 2013;48(5):247-55.

41. Yi CA, Lee KS, Lee HY, Kim S, Kwon OJ, Kim H, Choi JY, Kim BT, Hwang HS, Shim YM. Coregistered whole body magnetic resonance imaging-positron emission tomography (MRI-PET) versus PET-computed tomography plus brain MRI in staging resectable lung cancer: comparisons of clinical effectiveness in a randomized trial. Cancer. 2013;119(10):1784-91.

42. - Chandarana H, Heacock L, Rakheja R, Demello LR, Bonavita J, Block TK, Geppert C, Babb JS, Friedman KP. Pulmonary nodules in patients with primary malignancy: comparison of hybrid PET/MR and PET/CT imaging. Radiology. 2013. doi:10.1148/ radiol.13130620. Detection of lung nodules and their characterization is an important task in PET/CT. Identification of lesions in the lung is challenging with MRI compared with CT. This article gives insight into the performance of PET/MRI compared with PET/CT in this matter.

43. Heusch P, Buchbender C, Beiderwellen K, Nensa F, HartungKnemeyer V, Lauenstein TC, Bockisch A, Forsting M, Antoch G, Heusner TA. Standardized uptake values for $\left[{ }^{18} \mathrm{~F}\right]$ FDG in normal organ tissues: comparison of whole-body PET/CT and PET/MRI. Eur J Radiol. 2013;82(5):870-6.

44. Shrikhande SV, Barreto SG, Goel M, Arya S. Multimodality imaging of pancreatic ductal adenocarcinoma: a review of the literature. HPB (Oxford). 2012;14(10):658-68.

45. Francis IR. Pancreatic adenocarcinoma: diagnosis and staging using multidetector-row computed tomography (MDCT) and magnetic resonance imaging (MRI). Cancer Imaging. 2007;7(Spec Issue A): S160-5

46. Kauhanen SP, Komar G, Seppänen MP, Dean KI, Minn HR, Kajander SA, Rinta-Kiikka I, Alanen K, Borra RJ, Puolakkainen PA, Nuutila P, Ovaska JT. A prospective diagnostic accuracy study of 18F-fluorodeoxyglucose positron emission tomography/ computed tomography, multidetector row computed tomography, and magnetic resonance imaging in primary diagnosis and staging of pancreatic cancer. Ann Surg. 2009;250(6):957-63.

47. Lin JL, Barthel JS, Keshishian J, Eikman EA, Klapman JB. Negative predictive value of positron emission tomography/ computed tomography in patients with a clinical suspicion of pancreatic cancer. Pancreas. 2011;40(5):653-6.

48. Takanami K, Hiraide T, Tsuda M, Nakamura $Y$, Kaneta T, Takase K, Fukuda H, Takahashi S. Additional value of FDG PET/CT to contrast-enhanced CT in the differentiation between benign and malignant intraductal papillary mucinous neoplasms of the pancreas with mural nodules. Ann Nucl Med. 2011; 25(7):501-10.

49. Kim MJ, Lee KH, Lee KT, Lee JK, Ku BH, Oh CR, Heo JS, Choi $\mathrm{SH}$, Choi DW. The value of positron emission tomography/ computed tomography for evaluating metastatic disease in patients with pancreatic cancer. Pancreas. 2012;41(6):897-903.

50. Treglia G, Castaldi P, Rindi G, Giordano A, Rufini V. Diagnostic performance of Gallium-68 somatostatin receptor PET and PET/
CT in patients with thoracic and gastroenteropancreatic neuroendocrine tumours: a meta-analysis. Endocrine. 2012;42(1):80-7.

51. Nagamachi S, Nishii R, Wakamatsu H, Mizutani Y, Kiyohara S, Fujita S, Futami S, Sakae T, Furukoji E, Tamura S, Arita H, Chijiiwa K, Kawai K. The usefulness of 18F-FDG PET/MRI fusion image in diagnosing pancreatic tumor: comparison with 18F-FDG PET/CT. Ann Nucl Med. 2013. doi:10.1007/ s12149-013-0719-3.

52. Tatsumi M, Isohashi K, Onishi H, Hori M, Kim T, Higuchi I, Inoue A, Shimosegawa E, Takeda Y, Hatazawa J. 18F-FDG PET/ MRI fusion in characterizing pancreatic tumors: comparison to PET/CT. Int J Clin Oncol. 2011;16(4):408-15. doi:10.1007/s10147011-0202-x.

53. Niekel MC, Bipat S, Stoker J. Diagnostic imaging of colorectal liver metastases with CT, MR imaging, FDG PET, and/or FDG PET/CT: a meta-analysis of prospective studies including patients who have not previously undergone treatment. Radiology. 2010;257(3):674-84.

54. Lu YY, Chen JH, Chien CR, Chen WT, Tsai SC, Lin WY, Kao $\mathrm{CH}$. Use of FDG-PET or PET/CT to detect recurrent colorectal cancer in patients with elevated CEA: a systematic review and meta-analysis. Int J Colorectal Dis. 2013. doi:10.1007/s00384013-1659-z.

55. Lu YY, Chen JH, Ding HJ, Chien CR, Lin WY, Kao CH. A systematic review and meta-analysis of pretherapeutic lymph node staging of colorectal cancer by 18 F-FDG PET or PET/CT. Nucl Med Commun. 2012;33(11):1127-33.

56. Lubezky N, Metser U, Geva R, Nakache R, Shmueli E, Klausner JM, Even-Sapir E, Figer A, Ben-Haim M. The role and limitations of 18-fluoro-2-deoxy-D-glucose positron emission tomography (FDG-PET) scan and computerized tomography (CT) in restaging patients with hepatic colorectal metastases following neoadjuvant chemotherapy: comparison with operative and pathological findings. J Gastrointest Surg. 2007;11(4):472-8.

57. Drzezga A, Souvatzoglou M, Eiber M, Beer AJ, Fürst S, Martinez-Möller A, Nekolla SG, Ziegler S, Ganter C, Rummeny EJ, Schwaiger M. First clinical experience with integrated wholebody PET/MR: comparison to PET/CT in patients with oncologic diagnoses. J Nucl Med. 2012;53(6):845-55.

58. Kinkel K, Lu Y, Mehdizade A, Pelte MF, Hricak H. Indeterminate ovarian mass at US: incremental value of second imaging test for characterization-meta-analysis and Bayesian analysis. Radiology. 2005;236(1):85-94.

59. Sanli Y, Turkmen C, Bakir B, Iyibozkurt C, Ozel S, Has D, Yilmaz E, Topuz S, Yavuz E, Unal SN, Mudun A. Diagnostic value of PET/ CT is similar to that of conventional MRI and even better for detecting small peritoneal implants in patients with recurrent ovarian cancer. Nucl Med Commun. 2012;33(5):509-15.

60. Kubik-Huch RA, Dörffler W, von Schulthess GK, Marincek B, Köchli OR, Seifert B, Haller U, Steinert HC. Value of (18F)-FDG positron emission tomography, computed tomography, and magnetic resonance imaging in diagnosing primary and recurrent ovarian carcinoma. Eur Radiol. 2000;10(5):761-7.

61. Kim CK, Park BK, Choi JY, Kim BG, Han H. Detection of recurrent ovarian cancer at MRI: comparison with integrated PET/CT. J Comput Assist Tomogr. 2007;31(6):868-75.

62. Hoppe RT, Advani RH, Ai WZ, Ambinder RF, Aoun P, Bello CM, Bierman PJ, Blum KA, Chen R, Dabaja B, Duron Y, Forero A, Gordon LI, Hernandez-Ilizaliturri FJ, Hochberg EP, Maloney DG, Mansur D, Mauch PM, Metzger M, Moore JO, Morgan D, Moskowitz CH, Poppe M, Pro B, Winter JN, Yahalom J, Sundar $\mathrm{H}$, National Comprehensive Cancer Network. Hodgkin lymphoma, version 2.2012 featured updates to the NCCN guidelines. J Natl Compr Canc Netw. 2012;10(5):589-97.

63. Itti E, Meignan M, Berriolo-Riedinger A, Biggi A, Cashen AF, Véra P, Tilly H, Siegel BA, Gallamini A, Casasnovas RO, 
Haioun C. An international confirmatory study of the prognostic value of early PET/CT in diffuse large B-cell lymphoma: comparison between Deauville criteria and $\triangle$ SUVmax. Eur J Nucl Med Mol Imaging. 2013. doi:10.1007/s00259-013-2435-6.

64. Fuertes S, Setoain X, Lopez-Guillermo A, Carrasco JL, Rodríguez S, Rovira J, Pons F. Interim FDG PET/CT as a prognostic factor in diffuse large B-cell lymphoma. Eur J Nucl Med Mol Imaging. 2013;40(4):496-504.

65. Nievelstein RA, et al. Radiation exposure and mortality risk from CT and PET imaging of patients with malignant lymphoma. Eur Radiol. 2012;22(9): 1946-54.

66. Wu X, Kellokumpu-Lehtinen PL, Pertovaara H, Korkola P, Soimakallio S, Eskola H, Dastidar P. Diffusion-weighted MRI in early chemotherapy response evaluation of patients with diffuse large B-cell lymphoma-a pilot study: comparison with 2-deoxy2-fluoro-D-glucose-positron emission tomography/computed tomography. NMR Biomed. 2011;24(10):1181-90.

67. - Platzek I, Beuthien-Baumann B, Langner J, Popp M, Schramm G, Ordemann R, Laniado M, Kotzerke J, van den Hoff J. PET/ MR for therapy response evaluation in malignant lymphoma: initial experience. MAGMA. 2013;26(1):49-55. Lymphoma may be one of the future applications where PET/MRI may gain importance to reduce radiation exposure of patients with frequent repetitive imaging for follow-up of their disease. This article provides the first results on PET/MRI in lymphoma patients.

68. Punwani S, Taylor SA, Bainbridge A, Prakash V, Bandula S, De Vita E, Olsen OE, Hain SF, Stevens N, Daw S, Shankar A, Bomanji JB, Humphries PD. Pediatric and adolescent lymphoma: comparison of whole-body STIR half-Fourier RARE MR imaging with an enhanced PET/CT reference for initial staging. Radiology. 2010;255(1):182-90.
69. Kostakoglu L, Biggi A, Barrington S, Hutchings M, Meignan M, Gregianin M, Chauvies Gallamini A. Analysis of the Deauville criteria for the assessment of interim PET in advanced stage Hodgkin lymphoma patients enrolled in the IVS study part I: diagnostic accuracy and prognostic value. $J$ Nucl Med. 2012;53(Suppl 1):505.

70. Pfluger T, Melzer HI, Mueller WP, Coppenrath E, Bartenstein P, Albert MH, Schmid I. Diagnostic value of combined ${ }^{18} \mathrm{~F}-\mathrm{FDG}$ PET/MRI for staging and restaging in paediatric oncology. Eur $\mathrm{J}$ Nucl Med Mol Imaging. 2012;39(11):1745-55.

71. Kumar R, Xiu Y, Zhuang HM, Alavi A. 18F-fluorodeoxyglucosepositron emission tomography in evaluation of primary cutaneous lymphoma. Br J Dermatol. 2006;155(2):357-63.

72. Kumar J, Seith A, Kumar A, Sharma R, Bakhshi S, Kumar R, Agarwala S. Whole-body MR imaging with the use of parallel imaging for detection of skeletal metastases in pediatric patients with small-cell neoplasms: comparison with skeletal scintigraphy and FDG PET/CT. Pediatr Radiol. 2008;38(9):953-62.

73. Mueller WP, Melzer HI, Schmid I, Coppenrath E, Bartenstein P, Pfluger T. The diagnostic value of 18F-FDG PET and MRI in paediatric histiocytosis. Eur $\mathrm{J}$ Nucl Med Mol Imaging. 2013;40(3):356-63.

74. • Hirsch FW, Sattler B, Sorge I, Kurch L, Viehweger A, Ritter L, Werner P, Jochimsen T, Barthel H, Bierbach U, Till H, Sabri O, Kluge R. PET/MR in children. Initial clinical experience in paediatric oncology using an integrated PET/MR scanner. Pediatr Radiol. 2013;43(7):860-75. This article is one of the first to address PET/MRI in pediatric patients and describes the value and accuracy of PET/MRI in a pediatric oncologic patient population, addressing specific needs and operational challenges. 